\title{
Plant hormone transporters: what we know and what we would like to know
}

\author{
Jiyoung Park ${ }^{1 *}$, Youngsook Lee $^{2}$, Enrico Martinoia ${ }^{3}$ and Markus Geisler ${ }^{4^{*}}$
}

\begin{abstract}
Hormone transporters are crucial for plant hormone action, which is underlined by severe developmental and physiological impacts caused by their loss-offunction mutations. Here, we summarize recent knowledge on the individual roles of plant hormone transporters in local and long-distance transport. Our inventory reveals that many hormones are transported by members of distinct transporter classes, with an apparent dominance of the ATP-binding cassette (ABC) family and of the Nitrate transport1/Peptide transporter family (NPF). The current need to explore further hormone transporter regulation, their functional interaction, transport directionalities, and substrate specificities is briefly reviewed.
\end{abstract}

\section{Plants need many hormone transporters}

The hormone concept was coined in the beginning of the 20th century, although it had been already postulated by the Darwins based on their observation that phototropic bending of coleoptiles employs a spatial separation between the place of sensing in the shoot tip and the place of bending in the shoot below [1]. Today we know that what Darwin called 'certain influence' [1] is the phytohormone auxin, which is synthesized in the coleoptile tip, where light is sensed, and transported down to the appropriate site of action in the shoot [2].

Since then, many plant hormones have been identified and most have been found to be synthesized at different sites from their actions. Thus, it has become evident that hormones are transported, and consequently, hormone transporters are essential for precise regulation of plant growth and development by plant hormones. Now we understand that the proper integration of environmental

\footnotetext{
* Correspondence: j9park@ucsd.edu; markus.geisler@unifr.ch

${ }^{1}$ Division of Biological Sciences, University of California, San Diego, 9500 Gilman Drive, La Jolla, CA 92093-0116, USA

${ }^{4}$ Department of Biology, University of Fribourg, 1700 Fribourg, Switzerland Full list of author information is available at the end of the article
}

inputs with plant endogenous signaling requires the action of hormone transporting proteins. Evidence for this includes severe developmental and physiological impact that is caused by hormone transporter loss-of-function mutations: without transport, no correct hormone action.

Long distance transport has been demonstrated for many plant hormones, including auxins, abscisic acid (ABA), cytokinins, gibberellins (GAs), strigolactones, and salicylic acid. Hormones transported with the transpiration stream have to be loaded into the xylem and unloaded at the target cells. Similarly, hormones transported through the phloem may also require loading and unloading steps. Transporters involved in long distance transfer of auxin, ABA, cytokinins, and strigolactones have been reported, and further investigation would allow us to understand fully how individual transporters cooperate to achieve a systemic level of transport including via xylem or phloem. In cases where long-distance transport is achieved by cell-to-cell transport, such as for auxins, the highly coordinated action of import and export transporters at the contact surfaces of neighboring cells is needed. For local action of hormones (paracrine-like in animals) in some tissues such as seeds, short-distance transport between cells is sufficient, which could be carried out by exporter and importer proteins in adjacent cells (for example, see "Abscisic acid" section). Furthermore, in most cases plant hormones do not have only one target cell; therefore, several pairs of importers and exporters are required for the correct allocation and in order to guarantee the function of a complex hormonal network.

Only in a few cases has it been demonstrated that a plant hormone is synthesized in the same cells where its function is required, and these may not require intercellular transport mechanisms. A well-documented example regards ABA. Guard cells are able to synthesize ABA autonomously upon drought, thus rapidly closing stomata to prevent water loss [3]. Another case is ethylene, which is highly volatile and diffuses freely through lipid membranes. Therefore, ethylene synthesized at a specific 
location could be perceived by other cells far away from its site of synthesis as well as in the same cells without the need for a transporter [4]. However, it should be mentioned that the ethylene precursor ACC is mobile, and hence in this case it is not the hormone itself that is transported, but its precursor $[5,6]$.

Several hormones such as auxin and ABA are weak acids and hence partially present in their protonated forms in physiological $\mathrm{pH}$ conditions, which can diffuse quite easily through membranes $[7,8]$. Therefore, originally it has been postulated that transport of auxin and ABA from the apoplast into cells would occur by diffusion through the plasma membrane. Nowadays we know that diffusion of these hormones over the plasma membrane plays only a minor role in vivo and that transporters are required for proper delivery of auxin and ABA (see "Auxin" and "Abscisic acid" sections for details).

In this review, identification of plant transporters implicated in hormone transport and their individual and overlapping roles will be discussed. The transporters range from primary active pumps belonging to the ATP binding cassette $(\mathrm{ABC})$ transporter family (which couple hormone translocation to direct ATP hydrolysis); antiporters and symporters from the NITRATE TRANSPORTER (NRT) and Multidrug and toxic compound extrusion (MATE) transporter families (which use the proton motive force to create hormone concentration gradients); and facilitators of the SWEET family. This review will highlight how hormone transporters have been initially identified and-if so-how their individual activities and their substrate specificities have been verified. This would be crucial because deducing transport activities from protein sequence alignments or mutant phenotypes can have many pitfalls. For example, large substrate ranges of $\mathrm{ABC}$, NRT, and MATE transporter families make it difficult to predict a specific function of a transporter in hormone transport without adequate biochemical analyses. In cases where a protein of interest functions as a modulator of other transporters, it is necessary to carefully interpret transport activities/mutant phenotypes to elucidate functions of the modulator itself. Furthermore, phenotypes of transporter mutant plants need to be carefully examined since they may reflect secondary effects, such as changes in carbohydrate or nitrogen nutrition. It should also be mentioned that, although most studies have used plants carrying stable mutations in transporter genes to investigate functions of the transporters, it cannot be excluded that the observed phenotypes may be due to an adaptation of the plant to a specific mutation. In conclusion, it is always necessary to integrate multiple sources of evidence to demonstrate hormone transport by a transporter.

Finally, we will summarize what we would like to know about hormone transporters in the near future with respect to their discrete regulation, functional interaction, transport directionality, and substrate specificity.

\section{What we know about plant hormone transporters Auxins}

Natural auxins represent a surprisingly heterogeneous group of small aromatic carboxylic acids that act as a main coordinative signal for virtually all plant growth and development processes [9]. The most abundant auxin, IAA (indole-3-acetic acid), controls transcriptional regulation of multiple developmental processes via activation of a nuclear co-receptor complex (for a review, see [9]) but seems to regulate plant performance also on the post-transcriptional level [10].

Auxin responses depend on local auxin concentrations that are created by combinations of auxin biosynthesis, metabolism, and transport [7, 9]. In higher plants, IAA is translocated by two pathways: first, a fast, nondirectional passive transport through the phloem vasculature from biosynthetically active source tissues (such as young shoots) towards sink tissues (such as the root) [7]. Second, a slow, directional cell-to-cell transport of IAA, usually referred to as polar auxin transport (PAT), that is provided by active movement of IAA over the plasma membrane by auxin transporter proteins [9]. The directional, cell-to-cell transport is also possible for other plant hormones (such as jasmonic acids or strigolactones [11]), but has not yet been clearly demonstrated [12]. During PAT, local auxin minima and maxima, which are thought to be responsible for many developmental cues, are established, maintained, and fine-tuned by independent and combined action of members of at least three major families of auxin transporters [13] (Fig. 1): AUXIN1-RESISTANT1 (AUX1)/LIKE AUX1 (hereafter AUX1/LAX), PIN-FORMED proteins (hereafter PINs), and members of the B subfamily of ATP binding cassette $(\mathrm{ABC})$ transporters (hereafter $\mathrm{ABCBs)}$ $[14,15]$. While the $\operatorname{aux} 1$ and pin1 mutants were first identified in genetic screens for auxin (2,4-D) resistance [16] and developmental (flower) phenotypes [17], respectively, $A B C B 19 / M D R 1 / P G P 19$ was originally isolated in a screen for genes differentially expressed in response to the anion channel blocker NPPB (5-nitro-2(3-phenylpropylamino)-benzoic acid) [18]. Meanwhile, different $a b c b$ mutants were also identified in classic genetic screens $[19,20]$. For the key members of all three families, solid experimental data have been provided that characterize them as bona fide auxin transporters and their substrate specificities have been examined (reviewed in [13]).

Because IAA is a weak acid $(\mathrm{pKa}=4.75)$, a portion of it is lipophilic in its protonated form at apoplastic $\mathrm{pH}$ $(\approx 5.5)$; nevertheless, cellular uptake across the plasma membrane was shown to be dependent on AUX1/LAX 


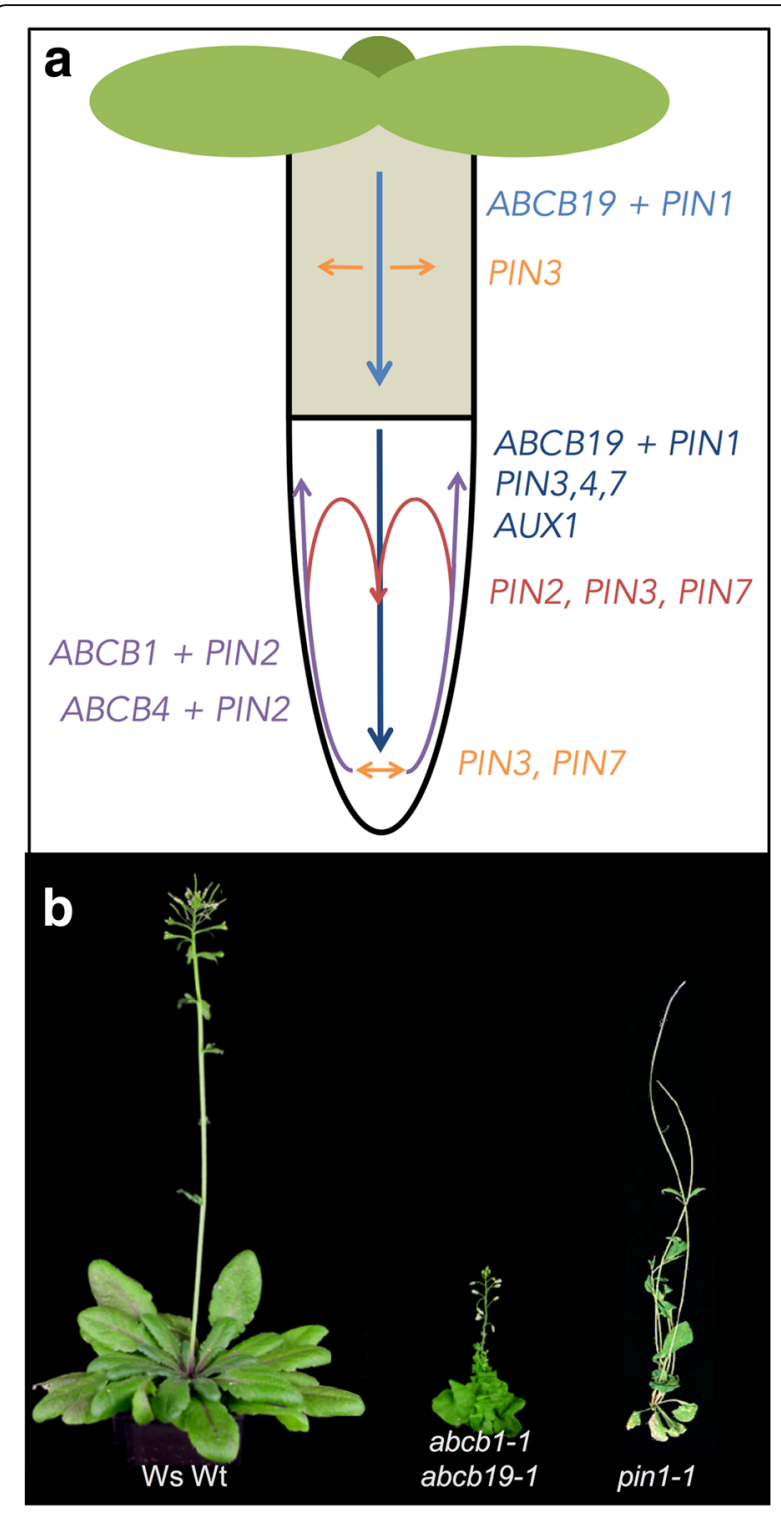

Fig. 1. Main auxin transport routes and transporters in Arabidopsis seedlings and growth phenotypes of adult, mutant plants lacking auxin exporters. a Contribution of ABCB-, AUX1/LAX-, and PIN-type transporters in main auxin (IAA) transport routes (arrows) of seedling roots and hypocotyls, where auxin research has been focused. Concerted actions indicating a shared, overlapping function of two (or more) PIN- and ABCB-type auxin exporters (for details, see [12]) are designated by color-coded arrows. Figure modified from [12]. b Growth phenotypes of soil-grown plants defective in auxin export From left to right: wild type (ecotype Wassilewskija, Ws), abcb1-1/ abcb19-1 (taken from [36], and reprinted by permission of the publisher, Taylor \& Francis Ltd, http://www.tandfonline.com) and pin1-1 (taken from [154] with permission). Note that, in contrast to the abcb1 abcb19 double mutant, abcb1 and abcb19 single mutants reveal only very subtle growth phenotypes, suggesting-despite opposite main transport routes-complementary action by $A B C B 19$ and $A B C B 1$ isoforms, respectively. Also note that other single PIN and higher order PIN mutants (without PIN1) [28] as well as single AUX1/LAX mutants and the quadruple auX1/lax mutant [23] reveal only very subtle growth phenotypes proteins functioning as high-affinity auxin-proton symporters [21, 22]. AUX1 has roles in both the acropetal auxin stream in the vascular cylinder as well as in auxin exclusion from the lateral root cap in the basipetal auxin stream (Fig. 1). In protophloem root cells, AUX1 reveals an asymmetric plasma membrane polarity at the shootward facing polar domain. Members of this family form a small gene family of four isoforms in Arabidopsis and regulate different developmental processes; however, the quadruple aux1/lax mutant reveals only very subtle growth phenotypes, including phyllotactic patterning defects [23].

An additional auxin uptake mechanism has been described with NITRATE TRANSPORTER 1.1 (NRT1.1), which is suggested to act as a dual function auxin transporter/nitrate transceptor under nitrogen starvation conditions [24, 25].

According to the chemiosmotic hypothesis [26, 27], auxin efflux may be a rate-limiting step in PAT [13] and efflux transporters of the PIN and the ABCB families are current candidate transporters for providing this activity. PINs, named after the pin-formed phenotype of the pin1 loss-of-function mutant (Fig. 1), are permease-like auxin transporters. PIN transporters can be classified into "long PINs" and "short PINs" based on the presence of an extensive hydrophilic loop separating two transmembrane domains. In Arabidopsis, long PINs (PIN1, 2, 3, 4, and 7) function as plasma membrane exporters, while short PINs (PIN5, 6, and 8) localize to endomembrane structures [13]. Most PINs, especially PIN1 and PIN2, exhibit a remarkable polar localization aligning well with their proposed roles in PAT, which has resulted in the concept that long PINs provide the vectorial component during PAT. As such they are thought to shape the socalled "reflux loop" in the root tip [28], controlling various developmental processes.

Unlike PINs and AUX1/LAX proteins, a subgroup of $\mathrm{ABCBs}$ act as primary active auxin pumps that are able to transport against steep auxin gradients [9, 14, 29]. Currently, ABCB isoforms, ABCB1, 4, 14, 15, 19, and 21, have been associated with PAT; however, only for $\mathrm{ABCB} 1,4,19$, and 21 have transport activities been tightly confirmed [29-34]. Interestingly, whereas ABCB1 and $\mathrm{ABCB} 19$ were shown to function as specific auxin exporters, Arabidopsis ABCB4 and 21 [29, 30, 33, 34] and Oryza sativa ABCB14 [35] were suggested to function as facultative IAA importers/exporters [30].

In correlation with their identity as auxin pumps, ABCBs are mainly expressed in meristematic tissues (both root and shoot), which contain high auxin concentrations $[29,36]$. These roles are on one hand in agreement with Arabidopsis mutant phenotypes for abcb19 and $a b c b 1 a b c b 19$, showing epinastic leaves and dwarfism as expected for an excess of apical auxin [18, 37]. 
On the other hand, phenotypes are also in line with gradual reductions in auxin transport; the $a b c b 1 a b c b 19$ mutant showed a $70 \%$ reduction in polar auxin transport [38]. In summary, $\mathrm{ABCBs}$ contribute much to polar transport of auxin (Fig. 1) despite their non-polar expression pattern [29].

Short PINs (PIN5, 6, 8) and members of a recently discovered family of PIN-LIKE (PILS) transporters were shown to function as auxin transporters on endomembrane (mainly endoplasmic reticulum (ER)) structures [39-41]. Their involvement in PAT is unclear; instead they were suggested to play roles in cellular auxin homeostasis by transporting auxin away from the nuclear auxin receptor. In another endomembrane, a tonoplast importer, WALLS ARE THIN 1 (WAT1), was shown to retrieve auxin from vacuoles, suggesting vacuoles function as an additional auxin compartment that might contribute to auxin homeostasis [42].

Several studies reported that another native auxin, indole-3-butyric acid (IBA), is also transported in a polar fashion by employing IAA-independent transport systems [43-46]. IBA hypersensitivity of mutants defective in $A B C G 36 / P D R 8 / P E N 3[46,47]$ and $A B C G 37 / P D R 9 /$ PIS1 [45, 46, 48] suggests that IBA is a common substrate exported by both ABCG36 and ABCG37, but IBA transport activity has only been examined for ABCG37 so far [45]. The roles of the two transporters in the putative apical or basal polar IBA transport are as yet unclear since they exhibit an outward-facing lateral polar localization, instead of an apical or basal polar localization expected for contributors to polar transport, in the root epidermis.

The $\mathrm{D}$ subfamily member of $\mathrm{ABC}$ transporter, PEROXISOMAL ABC TRANSPORTER1 (PXA1/ABCD1/ PED3/COMATOSE (CTS)), seems to be essential for full IBA responsiveness [49-51]. However, in contrast to fatty acyl-CoAs, IBA transport activity of ABCD1 has not yet been tested and abcd 1 mutant plants exhibit pleiotropic phenotypes, which may indicate wider substrate specificity for ABCD1 (see "Jasmonic acid" section).

In summary, in light of a pivotal role of auxin for plant performance, it does not come as a surprise that transport of auxin is distributed amongst several transporter families. While for key members of each transporter class auxin transport has been demonstrated biochemically, the contributions of individual family members in a complex auxin transport system are still not well understood.

\section{Abscisic acid}

Abscisic acid (ABA) is important for plant responses to environmental stresses such as drought and to pathogen infection, and for the regulation of developmental processes, including germination [8, 52, 53]. When plants are exposed to water deficiency, synthesis of ABA increases greatly. Perception of ABA by receptor proteins PYRABACTIN RESISTANCE (PYR)/PYR-LIKE (PYL)/REGULATORY COMPONENTS OF ABA RECEPTOR (RCAR) in the nucleus initiates a series of signal transduction steps involving the early signaling components PROTEIN PHOSPHATASEs 2C (PP2Cs) and SUCROSE NON-FERMENTING-1-RELATED PROTEIN KINASEs 2 (SnRK2s), which enable plants to cope with drought stress [54]. The most extensively studied ABA-induced response to drought is stomatal closing $[55,56]$. For a long time, roots were suspected to be the place of ABA synthesis upon drought $[8,57]$ in order to close stomata since, in nature, roots are the first site where the drought signal is perceived. Then ABA or its inactive form was postulated to move to the shoot via the vasculature. However, more recent reports suggest that drought-induced ABA synthesis occurs in shoot vasculature $[58,59]$. Consistent with this observation, the rate-limiting enzyme for $\mathrm{ABA}$ synthesis, 9-cisepoxycarotenoid dioxygenase 3 (NCED3), was induced in cells in vasculature in response to drought [60]. Recently, guard cells were also shown to produce ABA autonomously under low humidity [3]. A proportion of the weak acid ABA ( $\mathrm{pKa}=4.7)$ could diffuse into the plasma membrane from apoplastic spaces under normal conditions. However, as apoplastic $\mathrm{pH}$ increases under drought stress [61], the amount of the freely permeable form of ABA decreases. Therefore, ABA transporters are necessary to transfer ABA across the plasma membrane from the sites of synthesis (vascular tissues) to the sites of action (the guard cells), especially under drought conditions when ABA biosynthesis is induced.

G-subfamily ATP-binding cassette $(\mathrm{ABC})$ transporters ABCG25/WBC25 and ABCG40/PDR12 were reported to transport ABA over the plasma membrane out of vascular cells to the xylem, and into guard cells, respectively [62-64] (Fig. 2). ABCG25 is expressed mainly in phloem companion cells [64]. Its identity as an $\mathrm{ABA}$ exporter was demonstrated by transport activity assays using radiolabeled $\mathrm{ABA}$ and membrane vesicles isolated from insect cells expressing ABCG25 [63]. Also, ABCG25expressing Xenopus oocytes accumulated lower ABA than the control oocytes over time, when incubated in an ABA-containing bath solution [65]. ABA uptake activity of ABCG40 was demonstrated in yeast cells, a tobacco Bright Yellow 2 (BY2) cell line expressing ABCG40, and mesophyll protoplast cells of loss-offunction abcg 40 mutant plants using radiolabeled ABA [62]. abcg40 mutant plants exhibited impaired stomatal closing movement in response to ABA, which supported its role in ABA uptake into guard cells [62]. Together, these reports supported roles of the two ABCG 


\section{a Leaf exposed to drought}

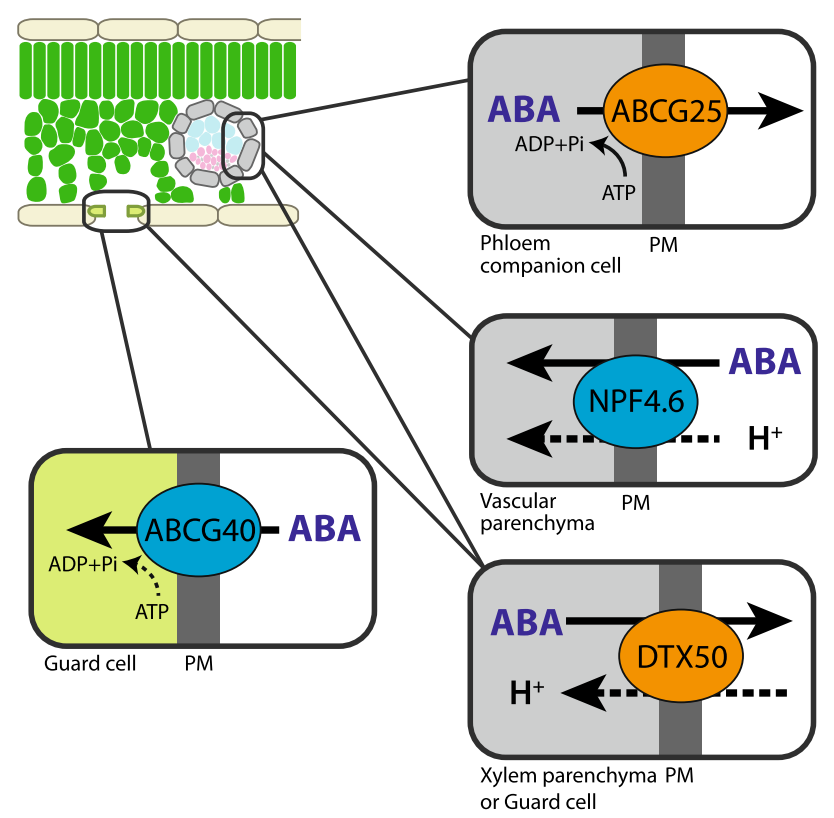

b Dormant seed

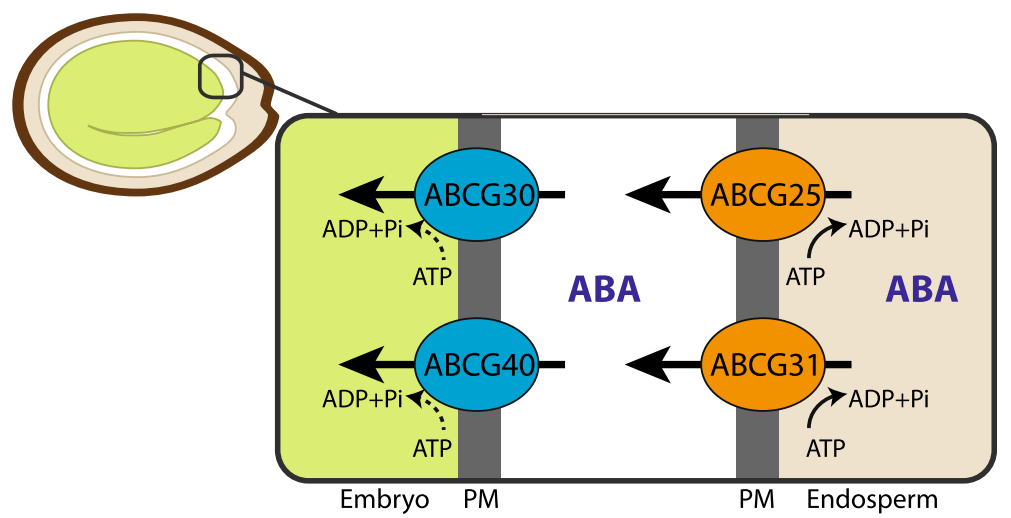

Fig. 2. ABA transporters in the leaf exposed to drought and in the dormant seed. Transporters that mediate efflux of ABA are marked in orange, and transporters that mediate influx of ABA are marked in blue. a When exposed to drought, ABA synthesized in vascular parenchymal cells is exported out of the cells via ABCG25 and DTX50. NPF4.6/AIT1 is reported to regulate the level of ABA in the vascular parenchymal cells. In the guard cell, ABCG40 takes up $A B A$, which induces stomatal closing. $\mathbf{b}$ Concerted action of $A B C$ transporters mediates $A B A$ transfer from endosperm to embryo, thus maintaining seed dormancy. At the endosperm cells, ABCG25 and ABCG31 export ABA to the extracellular space. ABCG30 and ABCG40 take up ABA into embryo cells. Under normal conditions, most ABA inside the plant cell (pH around 7.5) is in anionic forms, while in the apoplast at a pH between 5 and 6 a larger part of ABA is present in uncharged forms. Under drought conditions when the pH of the xylem sap increases, more ABA in the apoplast becomes charged. Whether ABC transporters that mediate ABA import use ATP hydrolysis as an energy source is not clear (dashed arrows). A driving force for DTX50 and NPF4.6 was not examined, but it is most likely dependent on proton motive force, similarly to many MATE transporters and NPF family transporters (dashed arrows). PM plasma membrane

transporters, ABCG25 and ABCG40, in supplying ABA from vascular cells to guard cells, thereby inducing stomatal closure under drought (Fig. 2).

In addition, ABCG25 and ABCG40, as well as two other Arabidopsis ABCG-type transporters, ABCG30 and ABCG31, were found to transport ABA from the endosperm of seed coats to the embryo, thus maintaining seed dormancy [66] (Fig. 2). Using germination assays with seed coats and embryos from different genotypes, involvement of the ABCG transporters in seed dormancy was examined [66]. Expression profiles and transport activity assays support that ABCG25 and ABCG31 catalyze the export of ABA from the endosperm, while ABCG40 and ABCG30 are responsible for 
the uptake of ABA into the embryo. However, transport assays using radiolabeled ABA revealed that the endosperm of the $a b c g 25 a b c g 31$ double loss-of-function plants still maintained at least $60 \%$ of the transport activity [66]. The half-size ABC transporter ABCG22 is implicated in drought stress tolerance; however, the ABA transport activity of ABCG22 was not detected in independent systems, which leaves the identity of AtABCG22 as an additional ABA transporter open [67].

DTX50, a transporter of the Multidrug and toxic compound extrusion (MATE) type transporter family [68], and NITRATE TRANSPORT1/PEPTIDE TRANSPORTER FAMILY (NPF) 4.6/NRT1.2/ABA-IMPORTING TRANSPORTER (AIT) 1, a member of the NPF transporters, have been shown to transport ABA [69] (Fig. 2). DTX50 is expressed in vascular tissues and localized at the plasma membrane. Its ABA efflux activity was examined in DTX50-expressing Escherichia coli cells and Xenopus oocytes, and mesophyll protoplasts of the $d t \times 50$ loss-of-function mutants using radiolabeled ABA. dtx50 mutant plants were hypersensitive to $\mathrm{ABA}$ in germination, root growth, and stomatal closing [68], altogether suggesting the involvement of DTX50 in ABA-related developmental processes. NPF4.6/AIT1 was discovered in a screen for proteins that induce ABA-dependent interaction between the ABA receptor PYR1 and PP2C ABI1 in a yeast two-hybrid ( $\mathrm{Y} 2 \mathrm{H})$ assay [69]. Transport assays and subsequent LC-MS/MS analysis supported the ABA uptake activity of NPF4.6/AIT1 in yeast. Loss-of-function mutants of NPF4.6/AIT1 were less sensitive to exogenously applied ABA than the wild type, exhibiting a decreased inhibition of seed germination [69]. Using the same $\mathrm{Y} 2 \mathrm{H}$ assay, other NPF members were also isolated as having potential ABA transport activity $[69,70]$, although their in planta functions remain to be further investigated.

$\mathrm{ABA}$ is stored in its inactive storage form, $\mathrm{ABA}$-glucose ester (ABA-GE), in the vacuole and $E R$, and under stress conditions $\mathrm{ABA}-\mathrm{GE}$ is cleaved into $\mathrm{ABA}$ and $\mathrm{ABA}$ is released into the cytosol [8, 71-73]. Uptake of ABA-GE into vacuoles has been shown to be catalyzed by AtABCC2 and a so-far-unknown proton antiporter [74]. However, transporters that sequester ABA-GE into the ER and transporters that export $\mathrm{ABA}$ released by glucosidases from the ER and vacuole are as yet unknown.

In short, members of many different transporter families have been found to participate in transport of ABA to cells near and far from the cells, which synthesize the hormone. Still, we expect additional ABA transporters will be identified to play critical physiological functions.

\section{Cytokinin}

Cytokinins represent a heterogenous group of $\mathrm{N}^{6}$ substituted adenine derivative hormones that regulate plant growth and development, including cambial meristem activities [75]. Free-base cytokinins such as trans-zeatin $(t \mathrm{Z})$ and $\mathrm{N}^{6}-\left(\Delta^{2}\right.$-isopentenyl) adenine (iP), are considered active, while their nucleoside conjugates are not. Sugar conjugates including cytokinin Oglucoside are thought to be inactive storage forms [75]. Once cytokinin is perceived by receptor proteins at the plasma membrane, a phospho-relay is initiated from Arabidopsis His kinases (AHKs), which transfer phosphoryl groups to Arabidopsis His Phosphotransfer proteins (AHPs), and then to Arabidopsis response regulators (ARRs), thus resulting in transcriptional and posttranscriptional regulation [76].

Different types of cytokinins are synthesized in roots and shoots, respectively; root-derived $t \mathrm{Z}$-type cytokinins and shoot-derived iP-type cytokinins move acropetally and basipetally, respectively, through the xylem and phloem [77, 78]. However, the molecular identity of the factors involved in long-distance transport of cytokinins remained obscure for a long time. Recently, two independent laboratories identified a G-type $A B C$ transporter, ABCG14, as critical for acropetal transport activity of $t$ Z-type cytokinins [79, 80]. ABCG14 loss-offunction mutant plants exhibited growth retardation in shoots including small leaves and short inflorescence stems, and a reduced number of vascular bundles, rescued by exogenous supplementation of $t \mathrm{Z}$. Reciprocal grafting experiments between $a b c g 14$ mutant plants and isogenic wild-type plants revealed that the roots of abcg14 cause growth retardation [79, 80] (Fig. 3).

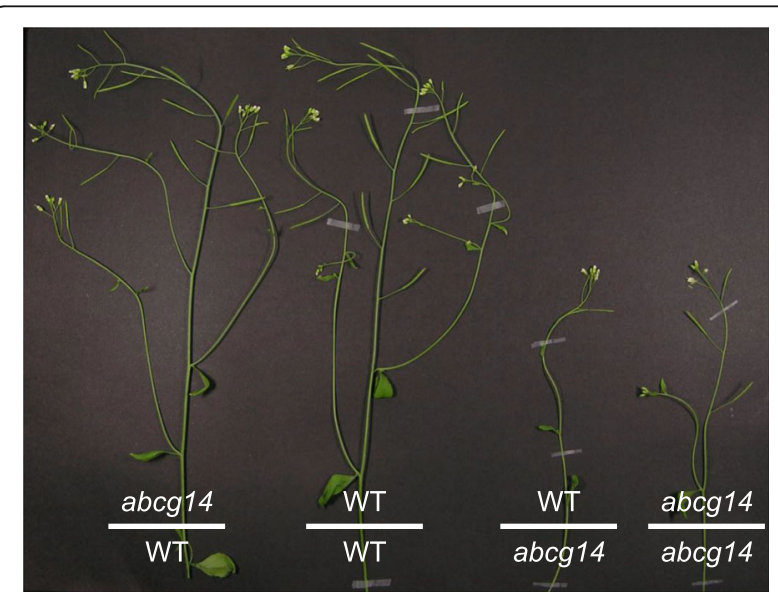

Fig. 3. Retarded growth phenotype of cytokinin transporter mutant abcg14. Reciprocal grafting experiments support a role of ABCG14 in root-to-shoot transport of cytokinin, which is crucial for normal growth and development of the shoot. The graft between an abcg14 mutant scion and wild-type rootstock (abcg14/WT) complemented the stunted growth of abcg14 loss-of-function mutant plants (as shown by the graft between abcg14 scion and rootstock (abcg14/abcg 14)), while the graft between a wild-type scion and abcg14 rootstock (WT/abcg14) maintained impaired growth. Image taken from [80] 
ABCG14 is expressed in cells of the root vasculature, including pericycle and procambial cells. Detailed cytokinin profiling in shoots and roots revealed that $t \mathrm{Z}$-type cytokinin concentrations were reduced in the shoot of abcg14 mutant plants [79, 80]. Finally, the role of ABCG14 in root-to-shoot transport of cytokinins was proven by translocation experiments using isotopelabeled $t Z$; abcg14 plants were found to accumulate less isotope-labeled $t \mathrm{Z}$ in their shoots when fed to the roots $[79,80]$. However, since ABCG14 has not been shown to transport cytokinin in any heterologous system, it is not known yet whether ABCG14 alone is sufficient for cytokinin transport or a partner protein is necessary for the function, and which kind of the many cytokinins is transported by ABCG14.

The equilibrative nucleoside transporter (ENT) family $[77,81,82]$ and the purine permease (PUP) family $[83,84]$ were also suggested to function as cytokinin transporters. The uptake transport activity of several ENT family members, including rice ENT2 and Arabidopsis ENT3, ENT6, ENT7, and ENT8, was shown upon expression in yeast using radio-labeled iP-riboside (iPR) and/or $t Z$-riboside $(t Z R)$ [77, 81, 82]. However, ent3 and ent 8 mutant plants exhibited only a marginal reduction in sensitivity to both iPR and $t$ ZR [82]; thus, their roles in cytokinin transport in planta remain obscure.

Recently, using a synthetic fluorescent reporter for cytokinin, PUP14 was isolated as a cytokinin uptake transporter at the plasma membrane and found to be important for normal development of the embryo in Arabidopsis [84]. Reducing PUP14 expression activated cytokinin signal transduction in the embryo and shoot apical meristem, while PUP14 over-expression led to inactivation of the pathway. The uptake activity of PUP14 was supported by radiolabeled $t \mathrm{Z}$ transport assays using mesophyll protoplasts expressing PUP14 and membrane vesicles isolated from PUP14-expressing tobacco plants. It was thus proposed that PUP14 takes up bioactive cytokinins from the apoplast and inhibits binding of cytokinins to plasma membrane-localized cytokinin receptors, suggesting PUP14 as a terminator of a cytokinin signal transduction [84]. This new concept for a transporter functioning in hormone depletion is, at first view, surprising since cytokinin receptors were recently shown to be enriched at the ER membrane [85]. Thus, deeper investigations would be required to establish further the proposed transporter-receptor interplay. In the case of other PUP members, such as PUP1 and PUP2, the uptake transport activity for $t \mathrm{Z}$ and iP was examined in yeast [83]; however, no genetic or biochemical evidence supporting their roles in planta has yet been provided. In short, an $\mathrm{ABC}$ transporter, $\mathrm{ABCG} 14$, and several ENT members have been shown to participate in cytokinin transport. Further biochemical analyses of ABCG14 and further analyses of in vivo functions of ENT members are necessary to establish a complete picture of cytokinin transport in plants.

\section{Gibberellins}

Gibberellins (GAs) regulate germination, growth-including shoot and root elongation-and reproductive development [86, 87]. They are a hormone group of tetracyclic diterpenoid acids labeled with "A numbers" in the order they were identified. Among more than 100 GAs, only a handful are known to be bioactive and recognized by the soluble receptor protein GIBBERELLIN INSENSITIVE DWARF 1 (GID1) and negative regulator DELLA proteins $[86,87]$. Reports using labeled GA provided physiological evidence that GA is translocated between different tissues ([88] and references therein). Recently, various strategies have allowed the identification of GA transporters.

Multiple members of the NPF family exhibit GA transport activities. A screen for mutant plants defective in root accumulation of fluorescein-tagged GA (GA-Fls) identified NPF3.1 as a putative GA transporter [89]. GA transport activity of NPF3.1 was examined in Xenopus oocytes using GA-Fls and GAs with subsequent LC-MS analyses. NPF3.1 was shown to import $\mathrm{GA}_{4}$ and, to a lesser extent, also $\mathrm{GA}_{1}$ and $\mathrm{GA}_{3}$ in a $\mathrm{pH}$-dependent manner, consistent with the proton motive forcedependence of the NPF transporter family. NPF3.1 lossof-function plants did not exhibit GA-related phenotypes [89], perhaps due to functional redundancy.

Based on a Y2H screen using the GA receptor GID1a and DELLA protein GA INSENSITIVE (GAI) as a readout, NPF4.1/AIT3 was shown to exhibit transport activities for $\mathrm{GA}_{1}, \mathrm{GA}_{3}$, and $\mathrm{GA}_{4}$ [70]. Also, using the same screening system, members of the sugar transporter SWEET family, AtSWEET13 and AtSWEET14, were identified to be involved in GA uptake [90]. Transport activities of SWEET13 and SWEET14 to $\mathrm{GA}_{3}$ and other GAs were further examined in yeast and Xenopus oocytes. SWEET-expressing yeast cells and Xenopus oocytes accumulated more GAs when measured by mass spectrometry. The sweet 13 sweet 14 double mutant, but not the respective single mutants, exhibited reduced fertility and delayed anther dehiscence, which is restored by addition of $\mathrm{GA}_{3}[90]$.

NPF2.10/GTR1 was reported for its role in transporting glucosinolates [91], defense compounds mainly found in the family Brassicaceae, but was also recently found to exhibit transport activities to GA and JA-Ile [92] (see also "Jasmonic acid" section). NPF2.10/GTR1 is involved in $\mathrm{GA}_{3}$ import, but not in the transport of the other bioactive GAs, $\mathrm{GA}_{1}$ and $\mathrm{GA}_{4}$, when examined in Xenopus oocytes [92]. NPF2.10/GTR1 loss-of-function plants exhibited a defect in stamen development, and 
$\mathrm{GA}_{3}$ rescued this phenotype, suggesting that the decreased fertility of $n p f 2.10 / \mathrm{gtr} 1$ is due to defects in GA transport [92] (Fig. 4). In summary, identification of many GA transporters was possible using novel screening strategies, which overcame the limitation of the conventional strategy of observing phenotypes of single gene knockout mutants. Such new screening methods are particularly important for plant hormone transporter research since there are likely many hormone transporters with similar and overlapping functions.

\section{Strigolactones}

Strigolactones were originally discovered as germination stimulants for parasitic weeds in the 1960s [93]. About 40 years after their discovery, scientists realized that

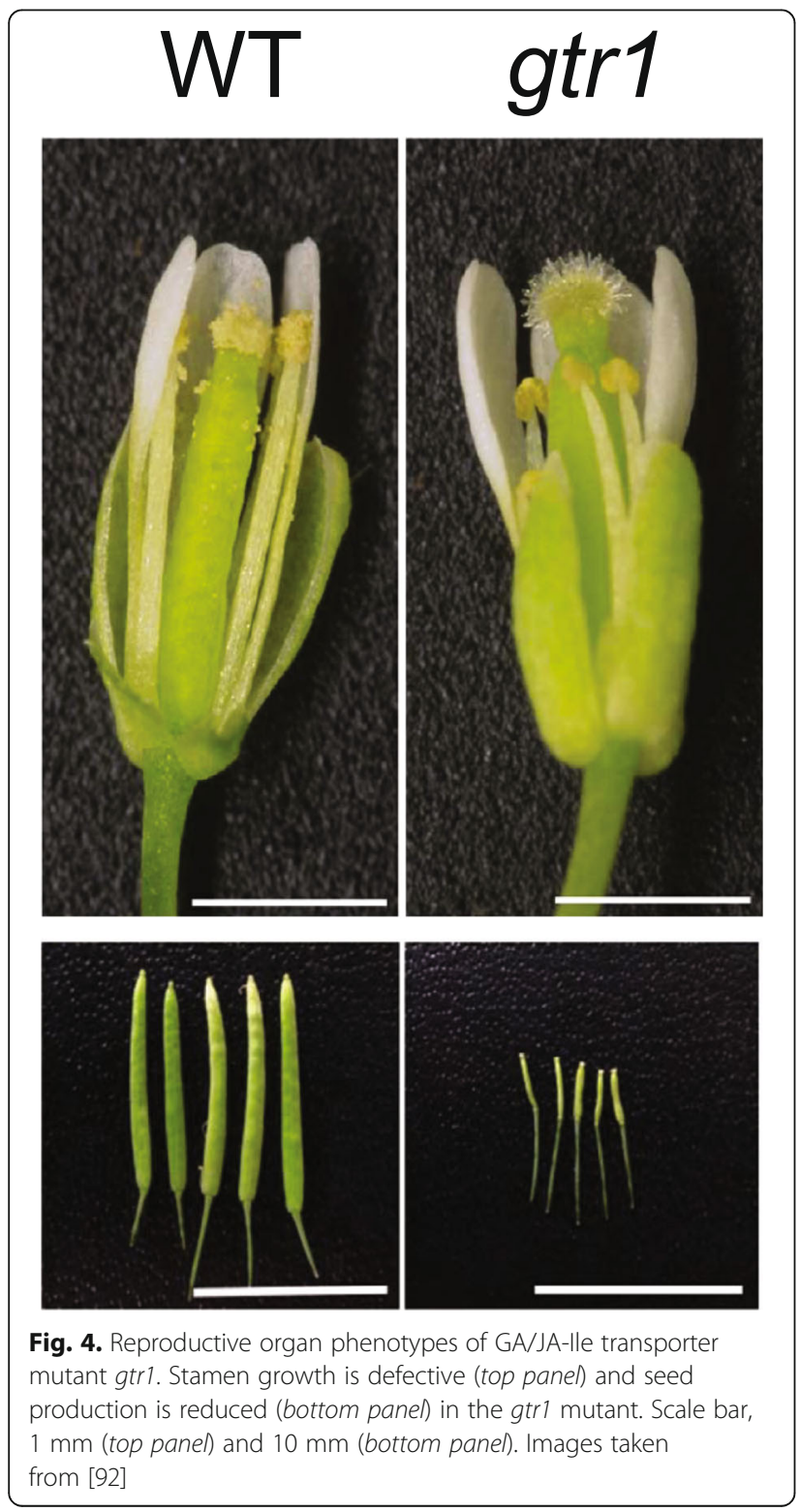

these carotenoid-derived compounds act both as signaling compounds inducing mycorrhizal symbiosis and phytohormones exhibiting intrinsic signaling functions. Within the plant, strigolactones act as inhibitors of lateral bud outgrowth and induce lateral root initiation and procambial division, which in principle overlap with auxin actions in developmental processes above- and below-ground. A plausible explanation for the overlap was found with the discovery that auxin transport was directly controlled by strigolactones [94]. In the presence of strigolactones, an F-box protein (MAX2 in Arabidopsis) interacts with an $\alpha / \beta$-hydrolase (D14 in Arabidopsis) and induces a downstream signaling cascade [95].

Strigolactones are produced both in roots and shoots, but grafting experiments have shown that root-derived strigolactones can rescue the shoot phenotype of a strigolactone biosynthesis mutant, indicating that strigolactones can be transported from the root to the shoot, most likely via the xylem [94]. However, whether a transporter is required for the root-to-shoot movement of strigolactones remained unknown until a recent report on the G-type ABC transporter, PDR1.

In a targeted approach, Kretzschmar et al. [96] identified PDR1 as a strigolactone transporter in Petunia. Petunia axillaris PDR1 (PaPDR1) was localized to the plasma membrane of the root tip and hypodermal passage cells, the latter being entry points of mycorrhizal fungi. The localization of PDR1 corresponds to the observation that strigolactones are excreted from roots to induce hyphal branching of mycorrhizal fungi. PDR1 loss-of-function plants excreted less strigolactone, were impaired in mycorrhization, and produced more branches in the aerial part of the plant. Further studies showed that the transfer of exogenously applied GR24, an artificial strigolactone, from the root to the shoot was impaired in PDR1 mutant plants, suggesting a role of PDR1 in root to shoot transport of strigolactones [96]. The dual role of PDR1 in transferring strigolactones to the shoot and to the rhizosphere is thought to be connected with its dual polar localization: in the root tip and the cortex the transporter is apically polar localized, while in hypodermal passage cells, PDR1 is localized laterally [97]. A subsequent study in tobacco showed that Nicotiana tabacum PDR6 (NtPDR6), a close homologue of PaPDR1, is also localized to hypodermal passage cells and that plants expressing antisense NtPDR6 were bushier than wild type, which is in line with a reduced allocation of strigolactones to the shoot [98, 99]. In Arabidopsis, no PDR1 homolog or strigolactone transporter has been identified so far, despite the fact that root to shoot translocation of strigolactones has been shown in Arabidopsis [100]. This may be due to the fact that in Arabidopsis the strigolactone-like compound methyl carlactonoate performs strigolactone-attributed functions 
[101]. It will be interesting, therefore, to unveil the corresponding transporter for methyl carlactonoate in Arabidopsis, including investigation of whether this transporter shows polar localization. Strigolactone transporters are the rare examples of hormone transporters first identified from non-Arabidopsis plants, and they seem to be important for plant interactions with other plants and microbes in the environment. Thus, it would be interesting to study evolution of the strigolactone transporters-how they diversified during establishment of parasitism and symbiosis.

\section{Jasmonates}

Jasmonates (JAs), the major component of the jasmine scent, are plant hormones important for reproductive development, wound responses, and plant immune signaling, especially against herbivores [102, 103]. JAs are produced via the oxidative metabolism of polysaturated fatty acids. JA biosynthesis is initiated in chloroplasts and plastids, and the subsequent steps occur in the peroxisomes. An amino acid conjugate of JAs, jasmonoylisoleucine (JA-Ile), is considered as the major bioactive form of JAs at the molecular level. JA-Ile is recognized by the nuclear receptor CORONATINE INSENSITIVE 1 (COI1), which then mediates proteasome-dependent degradation of the negative regulator JASMONATEZIM-DOMAIN (JAZ) proteins [102]. Recent reports using grafting and isotope-labeling experiments suggest that JAs or JA precursors could translocate from wounded tissues to unwounded tissues [11, 104], supporting transporter-mediated translocation of JAs (also reviewed in [105]).

Several members of NPFs were suggested to function as JA transporters. Via a $\mathrm{Y} 2 \mathrm{H}$ assay that detects JAdependent binding of the JA receptor COI1 and its interacting protein JAZ3, NPFs were tested for JA transport activities [70]. NPF4.1/AIT3 exhibited JA-Ile transport activity [70]; however, its in planta role in JA transport remains to be further elucidated due to potential redundancy with other JA transporters. Another NPF member, NPF2.10/GTR1, exhibited uptake activity for JA-Ile and JA when expressed in Xenopus oocytes [92]. NPF2.10/GTR1 also exhibits transport activities for other substrates including glucosinolates [91] and GA [92] (see "Gibberellin" section). GTR1 loss-of-function mutant was impaired in transferring JA and JA-Ile from wounded leaves to undamaged ones when isotope-labeled JA and JA-Ile were fed to the wounded leaves [106].

Synthesis of the precursor form of JA, 12-oxophytodienoic acid (OPDA), occurs in chloroplasts and plastids, while the final step of JA synthesis occurs in the peroxisome [102]. Chemical analyses and complementation of a yeast mutant support the idea that the peroxisomal D-type $\mathrm{ABC}$ transporter, $\mathrm{ABCD} 1 / \mathrm{CTS} /$ PXA1, mediates the transport of OPDA into the peroxisome and thus participates in JA biosynthesis [107-110]. When expressed in the yeast mutant lacking its homologs PXA1 and PXA2, functional AtABCD1 was targeted to yeast peroxisomes and complemented yeast that is defective in $\beta$-oxidation of fatty acids [110]. Basal levels of JA and wounding-inducible JA synthesis were reduced in $a b c d 1$ mutant plants [107, 109]. In addition, abcd1 mutant plants exhibit pleiotropic phenotypes, including enhanced seed dormancy, defects in seedling establishment in the absence of exogenous carbon source, and resistance to indole butyric acid (IBA) and 2,4-dichlorophenoxybutyric acid (2,4-DB), which suggest diverse roles of $\mathrm{ABCD} 1$ in mediating transport of fatty acylCoAs and IBA into the peroxisome for $\beta$-oxidation [107-109, 111] (also see "Auxin" section). Two barley $\mathrm{ABCD}$ proteins were suggested to act as homologs of Arabidopsis ABCD1; HvABCD2 was shown to complement Arabidopsis abcd1 mutant plants for OPDA sensitivity, whereas HvABCD1 complements yeast pxa1 pxa2 for $\beta$-oxidation of fatty acids [112], suggesting they have roles in OPDA transport and JA biosynthesis [112].

JA-Ile is recognized by the nuclear COI1/JAZ coreceptor complex, which reveals the necessity for nuclear entry of JA-Ile. AtABCG16/Jasmonate Transporter 1 (JAT1) exhibits a dual localization at the nuclear envelope and plasma membrane and was shown to mediate nuclear influx of JA-Ile and cellular efflux of JA across the plasma membrane [113]. JA transport activity of ABCG16/JAT1 was verified using isolated nuclei and suspension cells from ABCG16/JAT1 loss-of-function lines and in yeast cells pre-loaded with ${ }^{3} \mathrm{H}$-JA. Loss-offunction mutant abcg16/jat1 exhibited compromised JA signaling [113]. Recent identification of the transporters for intracellular transfer of JA and its precursor, as well as the JA transport GTR1, confirms the importance of coordinated distribution of JA and the precursors in the biosynthesis and function of JA.

\section{Salicylic acid}

Salicylic acid (SA) has been popular for its synthetic derivative aspirin, which is widely used for pain relief. SA is crucial for plants to invoke defense mechanisms against pathogens, especially plant innate immunity and systemic acquired resistance (SAR) [114]. Biosynthesis of SA occurs in the chloroplast, while its perception by receptor complexes is in the nucleus $[115,116]$. SA is also stored in the vacuole as an inactive form, SA O- $\beta$-glucoside (SAG) [114], altogether suggesting the necessity of transporters that mediate intracellular SA transport.

ENHANCED DISEASE SUSCEPTIBILITY5 (EDS5)/SA INDUCTION-DEFICIENT1 (sid1) was isolated from 
early screenings for enhanced pathogen susceptibility and defective SA accumulation after pathogen infection $[117,118]$. A recent report elucidated that EDS5 encodes a MATE-type transporter localized at the chloroplast envelope that exports SA from the chloroplast to the cytoplasm [119]. SA transport activity of EDS5 was demonstrated in isolated chloroplasts and heterologous yeast system incubated with radio-labeled SA [119], although more rigorous biochemical analysis will be needed to confirm that the direction of SA transport by EDS5 is out of the chloroplast.

Transport of an inactive storage form of SA, SA O- $\beta$ glucoside (SAG), into the vacuole is dependent on ATP and is thus suggested to require active transport, such as an ABC transporter-type mechanism in soybean [120, 121]. Also the well-known systemic distribution of SA or related mobile signals that induce systemic acquired resistance (SAR) ([114] and references therein) is dependent on a dedicated transport system. However, in both cases the identity of the transport systems has remained elusive.

\section{Ethylene}

Ethylene is a gaseous hormone involved in many physiological processes, including fruit ripening, senescence, abscission, and immune signaling, and also well known for crosstalk with other hormones including auxin and JA [122]. Ethylene is known to be freely diffusible through membranes and is thus able to move between cells and the intracellular space without transporter proteins [4]. However, the immediate precursor of ethylene, 1-aminocyclopropane-1-carboxylic acid (ACC), has been reported as a non-gaseous mobile signal, including longdistance transport through the xylem and the phloem $([5,6]$ and references therein).

$A C C$-resistant2 (ARE2) was isolated for insensitivity of the mutant plant to exogenously applied ACC, although its responses to ethylene were not altered [123]. ARE2 encodes for LYSINE HISTIDINE TRANSPORTER1 (LHT1), a member of the amino acid transporter family [123]. Transport assays using ${ }^{14} \mathrm{C}$-ACC in protoplasts from lht 1 mutant plants supported ACC uptake activity of a plasma membrane localized protein LHT1 [123]. LHT1 would thus provide a starting point to unveil the mechanism of ACC transport and the regulation of ethylene responses.

The ACC conjugate derivative N-malonylACC (MACC) is synthesized in the cytosol and transported into the vacuole for storage in an ATP-dependent manner [124]. The molecular identity of the MACC transporters still remains to be elucidated.

\section{Other hormones}

Brassinosteroids (BRs), with their well-known growthpromoting functions, have been thoroughly studied in terms of biosynthesis and signaling [125, 126]. Nevertheless, transport of BRs has not been unveiled much thus far. BRs are generally thought not to be transported between and within organs $[127,128]$. However, they are synthesized inside the cell, and their receptors are localized at the plasma membrane, exposing their ligandbinding sites to the extracellular space [125]. Therefore, the movement of BRs from the inside to the outside of the cell is necessary to initiate BR signal transduction and might thus-despite membrane permeability of these sterol compounds-require a plasma membrane exporter. Thus, it would be worth examining whether BR transport is mediated by transporter proteins.

Florigen is a hormone-like molecule responsible for inducing flowering, and its molecular identity was long sought after [129, 130]. Florigen is translocated from its site of synthesis, the leaf, to the shoot apex $[129,130]$. FLOWERING LOCUS T (FT) and FT-like proteins are suggested to fulfill the properties for florigen. They are produced in leaves and transported via the phloem to the shoot apical meristem of buds and growing tips [131]. Transport of FT into phloem companion cells was reported to be mediated by FT-INTERACTING PROTEIN1 (FTIP1) [132]. FTIP1 was shown to interact with FT in phloem companion cells and to control movement of FT from phloem companion cells to sieve elements, as supported by immunogold labeling of FT proteins in planta [132]. However, the transport mechanism for FTIP1 still remains elusive.

Small peptides are thought to have hormone-like activities that are involved in cell-to-cell signaling, and play crucial roles in plant growth and development, including defense mechanisms, the control of cell division and expansion, and pollen self-incompatibility [133]. Most of these small peptides are likely to be secreted to the apoplast, and thus transporters for these peptides might exist, especially when peptide hormones function in distal cells.

\section{What we would like to know about plant hormone transporters}

Our inventory of hormone transporters reveals that many hormones are transported by members of distinct transporter classes, such as primary active $A B C$ transporters, secondary active antiporters/symporters, and facilitators. What is remarkable is the apparent dominance of $\mathrm{ABC}$ transporters, mainly of the $\mathrm{B}$ and $\mathrm{G}$ subfamilies transporting auxins, $\mathrm{ABA}$, cytokinins, strigolactones, and $\mathrm{JA}$, and of the NPF transporting GA, ABA, and JA. However, we do not know yet why these groups of transporters are particularly more suitable for transport of plant hormones than other types. The reason may be that these transporter families accept chemically and structurally different classes of compounds and have 
evolved to recognize hormones. Another large transporter family transporting various substrates is the MATEs, for which transport of salicylic acid and ABA has been postulated. A large number of MATE transporters have not been functionally characterized yet, most likely due to their functional redundancy. Also, approximately 800 secondary transporters are predicted in Arabidopsis and rice and many of them await characterization [134].

At first glance it is surprising that one hormone can be imported or exported by several transporters, often belonging to different classes. For auxin transporters it has been suggested that their energization (primary vs. secondary active) correlates with different expression and polarity profiles and thus seems to match distinct functions in dependence with local hormone concentrations. Another aspect to be considered is that primary active transporters that are driven directly by ATP hydrolysis are likely to transport much smaller numbers of substrates per unit of time than the secondary active transporters. Thus, the primary active transporters may play a role in fine-tuning of hormone levels (as has been suggested for ABCB-type auxin transporters), while secondary active ones could function in mass transport of hormone molecules (as has been suggested for PINs), although this has not been demonstrated in detail for either auxin transport or other hormones. However, for auxin transporters, this possibility is supported by independent evolution of the different transporter families: ABCBs are evolutionarily conserved from ancient organisms, while PINs apparently appeared with the first land plants [135], arguing for involvement of PINs in the creation of more advanced plant architecture and performance. The appearance of land plants would have required the faster establishment of auxin gradients, as can only be provided by secondary active transporters (PINs) that usually show a higher turnover number. Interestingly, one study reported cooperative action of ABCBPIN pairs [12] resulting in synergistic (activation) or antagonistic (inhibition) interactions between the two types of auxin transporters [38]; it will be interesting to investigate if similar mechanisms are also found for other hormone transporters. Altogether, it appears that, in plants, concerted actions of distinct classes of hormone transporters-which function as fine-tuning transporters, mass transporters, and/or backup transporters-are a prerequisite for their appropriate function in hormone transport.

Interestingly, some members of B and G subfamilies of $\mathrm{ABC}$ transporters function as hormone importers. Arabidopsis ABCG30 and ABCG40 import ABA [62, 66], whereas $\mathrm{ABCB} 4$ and $\mathrm{ABCB} 21$ [30, 33, 34] and rice $A B C B 14$ [35] were suggested to function as auxin importers. This is surprising since most of the eukaryotic
$A B C$ proteins were found to be exporters [12]. Recently, evidence was provided that $\mathrm{ABCB} 4$ and $\mathrm{ABCB} 21$ are facultative IAA importers/exporters whose transport directionality is determined by intracellular auxin concentrations [30]. Obviously, any enzyme can function both ways; however, at the moment it is unclear if the hormone importers function as strict ATPases or as ATP-regulated channels. Mammalian ABCC7/CFTR (Cystic fibrosis transmembrane conductance regulator) was shown to require ATP for channel opening during chloride export [136].

With a few prominent exceptions, surprisingly little is known about the posttranscriptional regulation of hormone transporters in general. Establishment of PIN polarity has been studied extensively and is thought to be provided by dynamic, clathrin-mediated internalization [137]. PIN polarity is regulated by the combined action of the serine/threonine protein kinase of the AGC family, PINOID, and the protein phosphatase 2A, PP2A, which modulates the phosphorylation status of the hydrophilic PIN loop [138]. The plasma membrane presence of $\mathrm{ABCB} 1,4$, and 19 is dependent on the action of the FKBP42 protein, TWISTED DWARF1 (TWD1)/ ULTRACURVATA2 (UCU2), functioning as a chaperone during ER to plasma membrane delivery $[139,140]$. AUX1/LAX proteins appear to employ a distinct subcellular targeting mechanism from PIN and $\mathrm{ABCB}$ type auxin exporters [137, 141]. Recently, it was shown that lateral polarity of ABCG36 is dependent on ACTIN7 and the exocyst tethering complex [142]. Petunia PDR1 was reported to have dual polar localization: apically polar localized in the root tip and the cortex, and laterally localized in hypodermal passage cells [97]. In this respect, it is worth mentioning that despite directional delivery of many plant hormones, until now, polarized localization of hormone transporters has been demonstrated only for auxin and strigolactones. More detailed analyses might provide similar evidence for other hormone transporters which are involved in directional hormone transport.

Excitingly, some hormones seem to promote their own signal by stabilizing the plasma membrane presence of their transporters: in the case of ABCG25 [143] and PIN1 [144], localization of the proteins at the plasma membrane was found to be induced upon application of exogenous $\mathrm{ABA}$ and auxin, respectively. Despite the fact that underlying mechanisms of hormone-induced plasma membrane localization are different for ABCG25 (activation of recycling from early endosomes) and PIN1 (inhibition of endocytosis), it might be worth investigating whether plasma membrane stabilization of the transporters by the hormone substrates is a conserved phenomenon.

Protein phosphorylation regulates not only protein targeting of hormone transporters, but also their transport activity. Phosphorylation of cytoplasmic PIN loops by PINOID 
and related D6 protein kinases was recently shown to be essential for PIN transport activity [145]. Transport activity of $\mathrm{ABCB} 1$ and $\mathrm{ABCB} 19$ was also regulated by protein phosphorylation by PINOID, and the blue light receptor, PHOTOTROPIN1, respectively $[146,147]$. Transport activity of GTR1 in Xenopus oocytes was abolished when a threonine residue between transmembrane 3 and 4 was substituted to the phospho-mimicking aspartic acid, and it was suggested that de-phosphorylation of the threonine is important for dimerization of GTR1 and plasma membrane localization [148]. Whether protein phosphorylation involves regulation of other hormone transporters would be interesting to investigate in the future.

Another exciting finding is that plant hormone transporters, at least a subset of ABC-type hormone transporters, have a very high degree of substrate specificity in contrast to their mammalian orthologues: ABCG25 and ABCG40 seem to transport only the physiologically active ABA enantiomer, (S)-ABA, but not (R)-ABA [62, 63], while $A B C B 1$ is specific for a subset of auxins, including the synthetic, active auxin 1-NAA, but does not transport 2-NAA [149]. The molecular basis for this high specificity is unknown. However, kingdom-specific, putative auxin-binding motif clusters were recently found in the transmembrane domains of ABCB1 [150], which await biochemical characterization to confirm predicted function.

In contrast, some members of $\mathrm{ABC}$ transporters seem to have multi-substrate specificity: abcd1/cts mutant plants exhibit pleiotropic phenotypes, including increased seed dormancy, defects in seedling establishment without an additional carbon source, and resistance to IBA, 2,4-DB, and OPDA, which suggests diverse roles of $\mathrm{ABCD} 1$ in mediating transport of fatty acyl-CoAs, IBA, and OPDA into the peroxisome $[49,51,112,151]$. Further, ABCG36 and ABCG37 transport structurally unrelated auxinic compounds like IBA, 2.4-D, and NPA [45, 152], but not IAA [45], while ABCG36 seems to also transport the heavy metal cadmium [153] and indole glucosinolates [47]. Finally, NPF2.10/GTR1 has transport activities for diverse substrates, including $\mathrm{GA}_{3}$, JAs, and glucosinolates, which correspond to pleiotropic phenotypes of the lossof-function mutant plant [91, 92]. More rigorous biochemical verification of substrate specificities could clarify whether the proposed multi-substrate specificity can be safely attributed to individual transporters. In-depth biochemistry combined with physiological experiments would also uncover whether multi-specificity is linked to specific localization of the transporters in planta, or regulated by posttranslational modification and/or interacting partners.

\section{Conclusions}

In summary, it appears that we have learned a lot about plant hormone transport over past years by identifying individual roles of transporters and their impact on hormone physiology. This review underlines the central roles of hormone transporters for hormone action and thus proper plant growth, architecture, and survival. However, many questions remain to be answered about their discrete regulation, functional interaction, transport directionality, and substrate specificity. Finally, many hormones, like IAA and ABA, are demobilized and deactivated by conjugation. However, besides the physiological function and conversion of these conjugates, their transporters are mostly unknown.

\section{Acknowledgments}

This work was supported by grants from the Swiss National Funds to MG (31003A_165877) and EM (31003A-152831), a National Research Foundation (NRF) of Korea grant funded by the Ministry of Science, Information and Communication Technology, and Future Planning, Korea awarded to YL (NRF-2015R1A2A1A01004294), and the European Space Association (ESA) to MG (CORA-LIRAT). Research of JP was supported by a National Institutes of Health grant (GM060396) to Julian Schroeder (UC San Diego). We thank Drs. Takashi Kuromori and Edward Farmer for critically reading the manuscript.

\section{Authors' contributions}

$J P, Y L, E M$, and MG wrote the manuscript. All authors have read and agreed to the content.

\section{Competing interests}

The authors declare that they have no competing interests.

\section{Publisher's Note}

Springer Nature remains neutral with regard to jurisdictional claims in published maps and institutional affiliations.

\section{Author details}

'Division of Biological Sciences, University of California, San Diego, 9500 Gilman Drive, La Jolla, CA 92093-0116, USA. ²Division of Integrative Bioscience and Biotechnology, POSTECH, Pohang 37673, South Korea. ${ }^{3}$ Institute for Plant Biology, University of Zurich, Zollikerstrasse 107, 8008 Zurich, Switzerland. ${ }^{4}$ Department of Biology, University of Fribourg, 1700 Fribourg, Switzerland.

Published online: 25 October 2017

\section{References}

1. Darwin C, Darwin F. The power of movements in plants. London: Murray; 1880.

2. Christie JM, Murphy AS. Shoot phototropism in higher plants: new light through old concepts. Am J Bot. 2013;100:35-46.

3. Bauer H, Ache P, Lautner S, Fromm J, Hartung W, Al-Rasheid KA, et al. The stomatal response to reduced relative humidity requires guard cell-autonomous ABA synthesis. Curr Biol. 2013;23:53-7.

4. Davies PJ. The plant hormones: their nature, occurrence, and functions. In: Davies PJ, editor. The plant hormones: biosynthesis, signal transduction, action! 3rd ed. Dordrecht: Springer Netherlands; 2010. p. 1-15.

5. Yang SF, Hoffmann NE. Ethylene biosynthesis and its regulation in higher plants. Annu Rev Plant Physiol. 1984;35:155-89.

6. Van de Poel B, Van Der Straeten D. 1-aminocyclopropane-1-carboxylic acid (ACC) in plants: more than just the precursor of ethylene! Front Plant Sci. 2014:5:640.

7. Vanneste S, Friml J. Auxin: a trigger for change in plant development. Cell. 2009;136:1005-16.

8. Zeevaart JAD, Creelman RA. Metabolism and physiology of abscisic acid. Annu Rev Plant Physiol Plant Mol Biol. 1988;39:439-73.

9. Grones P, Friml J. Auxin transporters and binding proteins at a glance. J Cell Sci. 2015;128:1-7.

10. Zhu J, Geisler M. Keeping it all together: auxin-actin crosstalk in plant development. J Exp Bot. 2015;66:4983-98. 
11. Gasperini D, Chauvin A, Acosta IF, Kurenda A, Stolz S, Chetelat A, et al. Axial and radial oxylipin transport. Plant Physiol. 2015;169:2244-54.

12. Geisler M, Aryal, B., di Donato, M., and Hao, P. A critical view on ABC transporters and their interacting partners in auxin transport. Plant Cell Physiol. 2017;58:1601-14. doi:10.1093/pcp/pcx104.

13. Geisler M, Wang B, Zhu J. Auxin transport during root gravitropism: transporters and techniques. Plant Biol (Stuttg). 2014;16(Suppl 1):50-7.

14. Geisler M, Bailly A, Ivanchenko M. Master and servant: regulation of auxin transporters by FKBPs and cyclophilins. Plant Sci. 2016;245:1-10.

15. Kerr ID, Bennett MJ. New insight into the biochemical mechanisms regulating auxin transport in plants. Biochem J. 2007;401:613-22.

16. Maher EP, Martindale SJ. Mutants of Arabidopsis thaliana with altered responses to auxins and gravity. Biochem Genet. 1980;18:1041-53.

17. Okada K, Ueda J, Komaki MK, Bell CJ, Shimura Y. Requirement of the auxin polar transport system in early stages of Arabidopsis floral bud formation. Plant Cell. 1991;3:677-84

18. Noh B, Murphy AS, Spalding EP. Multidrug resistance-like genes of Arabidopsis required for auxin transport and auxin-mediated development. Plant Cell. 2001;13:2441-54

19. Zhao H, Liu L, Mo H, Qian L, Cao Y, Cui S, et al. The ATP-binding cassette transporter $\mathrm{ABCB} 19$ regulates postembryonic organ separation in Arabidopsis. PLoS One. 2013;8:e60809.

20. Ye L, Liu L, Xing A, Kang D. Characterization of a dwarf mutant allele of Arabidopsis MDR-like ABC transporter AtPGP1 gene. Biochem Biophys Res Commun. 2013;441:782-6.

21. Bennett MJ, Marchant A, Green HG, May ST, Ward SP, Millner PA, et al. Arabidopsis AUX1 gene: a permease-like regulator of root gravitropism. Science. 1996;273:948-50.

22. Swarup K, Benkova E, Swarup R, Casimiro I, Peret B, Yang Y, et al. The auxin influx carrier LAX3 promotes lateral root emergence. Nat Cell Biol. 2008;10: 946-54.

23. Bainbridge K, Guyomarc'h S, Bayer E, Swarup R, Bennett M, Mandel T, et al. Auxin influx carriers stabilize phyllotactic patterning. Genes Dev. 2008;22: 810-23.

24. Krouk G, Lacombe B, Bielach A, Perrine-Walker F, Malinska K, Mounier E, et al. Nitrate-regulated auxin transport by NRT1.1 defines a mechanism for nutrient sensing in plants. Dev Cell. 2010;18:927-37.

25. $\mathrm{Ho} \mathrm{CH}$, Lin $\mathrm{SH}, \mathrm{Hu}$ HC, Tsay YF. CHL1 functions as a nitrate sensor in plants. Cell. 2009;138:1184-94.

26. Rubery PH, Sheldrake AR. Carrier-mediated auxin transport. Planta. 1974;118: 101-21.

27. Raven JA. Transport of indoleacetic-acid in plant-cells in relation to $\mathrm{pH}$ and electrical potential gradients, and its significance for polar IAA transport. New Phytol. 1975;74:163-72.

28. Blilou I, Xu J, Wildwater M, Willemsen V, Paponov I, Friml J, et al. The PIN auxin efflux facilitator network controls growth and patterning in Arabidopsis roots. Nature. 2005:433:39-44.

29. Geisler M, Murphy AS. The ABC of auxin transport: the role of p-glycoproteins in plant development. FEBS Lett. 2006:580:1094-102.

30. Kamimoto Y, Terasaka K, Hamamoto M, Takanashi K, Fukuda S, Shitan N, et al. Arabidopsis ABCB21 is a facultative auxin importer/exporter regulated by cytoplasmic auxin concentration. Plant Cell Physiol. 2012;53:2090-100.

31. Santelia D, Henrichs S, Vincenzetti V, Sauer M, Bigler L, Klein M, et al Flavonoids redirect PIN-mediated polar auxin fluxes during root gravitropic responses. J Biol Chem. 2008:283:31218-26.

32. Bouchard R, Bailly A, Blakeslee JJ, Oehring SC, Vincenzetti V, Lee OR, et al. Immunophilin-like TWISTED DWARF1 modulates auxin efflux activities of Arabidopsis P-glycoproteins. J Biol Chem. 2006;281:30603-12.

33. Santelia D, Vincenzetti $V$, Azzarello E, Bovet $L$, Fukao $Y$, Duchtig $P$, et al. MDR-like ABC transporter AtPGP4 is involved in auxin-mediated lateral root and root hair development. FEBS Lett. 2005;579:5399-406

34. Terasaka K, Blakeslee JJ, Titapiwatanakun B, Peer WA, Bandyopadhyay A, Makam SN, et al. PGP4, an ATP binding cassette P-glycoprotein, catalyzes auxin transport in Arabidopsis thaliana roots. Plant Cell. 2005;17:2922-39.

35. Xu Y, Zhang S, Guo H, Wang S, Xu L, Li C, et al. OsABCB14 functions in auxin transport and iron homeostasis in rice (Oryza sativa L.). Plant J. 2014; 79:106-17.

36. Bailly A, Sovero V, Geisler M. The Twisted Dwarf's ABC: How immunophilins regulate auxin transport. Plant Signal Behav. 2006;1:277-80.

37. Geisler M, Kolukisaoglu HU, Bouchard R, Billion K, Berger J, Saal B, et al. TWISTED DWARF1, a unique plasma membrane-anchored immunophilin- like protein, interacts with Arabidopsis multidrug resistance-like transporters AtPGP1 and AtPGP19. Mol Biol Cell. 2003;14:4238-49.

38. Blakeslee JJ, Bandyopadhyay A, Lee OR, Mravec J, Titapiwatanakun B, Sauer $\mathrm{M}$, et al. Interactions among PIN-FORMED and P-glycoprotein auxin transporters in Arabidopsis. Plant Cell. 2007;19:131-47.

39. Ding Z, Wang B, Moreno I, Duplakova N, Simon S, Carraro N, et al. ER-localized auxin transporter PIN8 regulates auxin homeostasis and male gametophyte development in Arabidopsis. Nat Commun. 2012;3:941.

40. Mravec J, Skupa P, Bailly A, Hoyerova K, Krecek P, Bielach A, et al. Subcellular homeostasis of phytohormone auxin is mediated by the ER-localized PIN5 transporter. Nature. 2009:459:1136-40.

41. Barbez E, Kubes M, Rolcik J, Beziat C, Pencik A, Wang B, et al. A novel putative auxin carrier family regulates intracellular auxin homeostasis in plants. Nature. 2012;485:119-22.

42. Ranocha P, Dima O, Nagy R, Felten J, Corratge-Faillie C, Novak O, et al. Arabidopsis WAT1 is a vacuolar auxin transport facilitator required for auxin homoeostasis. Nat Commun. 2013;4:2625.

43. Rashotte AM, Poupart J, Waddell CS, Muday GK. Transport of the two natural auxins, indole-3-butyric acid and indole-3-acetic acid, in Arabidopsis. Plant Physiol. 2003;133:761-72.

44. Strader LC, Bartel B. Transport and metabolism of the endogenous auxin precursor indole-3-butyric acid. Mol Plant. 2011;4:477-86.

45. Ruzicka K, Strader LC, Bailly A, Yang H, Blakeslee J, Langowski L, et al. Arabidopsis PIS1 encodes the ABCG37 transporter of auxinic compounds including the auxin precursor indole-3-butyric acid. Proc Natl Acad Sci U S A. 2010;107:10749-53.

46. Strader LC, Bartel B. The Arabidopsis PLEIOTROPIC DRUG RESISTANCE8/ ABCG36 ATP binding cassette transporter modulates sensitivity to the auxin precursor indole-3-butyric acid. Plant Cell. 2009;21:1992-2007.

47. Lu X, Dittgen J, Pislewska-Bednarek M, Molina A, Schneider B, Svatos A, et al Mutant allele-specific uncoupling of PENETRATION3 functions reveals engagement of the ATP-binding cassette transporter in distinct tryptophan metabolic pathways. Plant Physiol. 2015;168:814-27.

48. Borghi L, Kang J, Ko D, Lee Y, Martinoia E. The role of ABCG-type ABC transporters in phytohormone transport. Biochem Soc Trans. 2015;43:924-30.

49. Zolman BK, Silva ID, Bartel B. The Arabidopsis pxa1 mutant is defective in an ATP-binding cassette transporter-like protein required for peroxisomal fatty acid B-oxidation. Plant Physiol. 2001;127:1266-78.

50. Hooks MA, Turner JE, Murphy EC, Johnston KA, Burr S, Jaroslawski S. The Arabidopsis ALDP protein homologue COMATOSE is instrumental in peroxisomal acetate metabolism. Biochem J. 2007;406:399-406.

51. Footitt S, Slocombe SP, Larner V, Kurup S, Wu Y, Larson T, et al. Control of germination and lipid mobilization by COMATOSE, the Arabidopsis homologue of human ALDP. EMBO J. 2002;21:2912-22.

52. Finkelstein RR, Gampala SSL, Rock CD. Abscisic acid signaling in seeds and seedlings. Plant Cell. 2002;14(Suppl 1):S15-45.

53. Lee SC, Luan S. ABA signal transduction at the crossroad of biotic and abiotic stress responses. Plant Cell Environ. 2012;35:53-60.

54. Cutler SR, Rodriguez PL, Finkelstein RR, Abrams SR. Abscisic acid: emergence of a core signaling network. Annu Rev Plant Biol. 2010;61:651-79.

55. Schroeder Jl, Allen GJ, Hugouvieux V, Kwak JM, Waner D. Guard cell signal transduction. Annu Rev Plant Physiol Plant Mol Biol. 2001;52:627-58.

56. Blatt MR. Cellular signaling and volume control in stomatal movements in plants. Annu Rev Cell Dev Biol. 2000;16:221-41.

57. Jackson MB. Are plant hormones involved in root to shoot communication? Adv Bot Res. 1993;19:103-87.

58. Christmann A, Hoffmann T, Teplova I, Grill E, Müller A. Generation of active pools of abscisic acid revealed by in vivo imaging of water-stressed Arabidopsis. Plant Physiol. 2005;137:209-19.

59. Christmann A, Weiler EW, Steudle E, Grill E. A hydraulic signal in root-toshoot signalling of water shortage. Plant J. 2007;52:167-74.

60. Endo A, Sawada Y, Takahashi H, Okamoto M, Ikegami K, Koiwai H, et al. Drought induction of Arabidopsis 9-cis-epoxycarotenoid dioxygenase occurs in vascular parenchyma cells. Plant Physiol. 2008;147:1984-93.

61. Wilkinson S, Davies WJ. Xylem sap pH increase: a drought signal received at the apoplastic face of the guard cell that involves the suppression of saturable abscisic acid uptake by the epidermal symplast. Plant Physiol. 1997;113:559-73.

62. Kang J, Hwang JU, Lee M, Kim YY, Assmann SM, Martinoia E, et al. PDR-type $\mathrm{ABC}$ transporter mediates cellular uptake of the phytohormone abscisic acid. Proc Natl Acad Sci U S A. 2010;107:2355-60. 
63. Kuromori T, Miyaji T, Yabuuchi H, Shimizu H, Sugimoto E, Kamiya A, et al. $A B C$ transporter AtABCG25 is involved in abscisic acid transport and responses. Proc Natl Acad Sci U S A. 2010;107:2361-6.

64. Kuromori T, Sugimoto E, Shinozaki K. Intertissue signal transfer of abscisic acid from vascular cells to guard cells. Plant Physiol. 2014;164:1587-92.

65. Xu D, Veres D, Belew ZM, Olsen CE, Nour-Eldin HH, Halkier BA. Functional expression and characterization of plant $A B C$ transporters in Xenopus laevis oocytes for transport engineering purposes. Methods Enzymol. 2016:576:207-24

66. Kang J, Yim S, Choi H, Kim A, Lee KP, Lopez-Molina L, et al. Abscisic acid transporters cooperate to control seed germination. Nat Commun. 2015;6:8113.

67. Kuromori T, Sugimoto E, Shinozaki K. Arabidopsis mutants of AtABCG22, an $A B C$ transporter gene, increase water transpiration and drought susceptibility. Plant J. 2011;67:885-94.

68. Zhang H, Zhu H, Pan Y, Yu Y, Luan S, Li L. A DTX/MATE-type transporter facilitates abscisic acid efflux and modulates ABA sensitivity and drought tolerance in Arabidopsis. Mol Plant. 2014;7:1522-32.

69. Kanno Y, Hanada A, Chiba Y, Ichikawa T, Nakazawa M, Matsui M, et al. Identification of an abscisic acid transporter by functional screening using the receptor complex as a sensor. Proc Natl Acad Sci U S A. 2012;109:9653-8.

70. Chiba Y, Shimizu T, Miyakawa S, Kanno Y, Koshiba T, Kamiya Y, et al. Identification of Arabidopsis thaliana NRT1/PTR FAMILY (NPF) proteins capable of transporting plant hormones. J Plant Res. 2015;128:679-86.

71. Lee KH, Piao HL, Kim HY, Choi SM, Jiang F, Hartung W, et al. Activation of glucosidase via stress-induced polymerization rapidly increases active pools of abscisic acid. Cell. 2006;126:1109-20.

72. Nambara E, Marion-Poll A. Abscisic acid biosynthesis and catabolism. Annu Rev Plant Biol. 2005:56:165-85.

73. $X u$ ZY, Lee KH, Dong T, Jeong JC, Jin JB, Kanno Y, et al. A vacuolar beta-glucosidase homolog that possesses glucose-conjugated abscisic acid hydrolyzing activity plays an important role in osmotic stress responses in Arabidopsis. Plant Cell. 2012;24:2184-99.

74. Burla B, Pfrunder S, Nagy R, Francisco RM, Lee Y, Martinoia E. Vacuolar transport of abscisic acid glucosyl ester is mediated by ATP-binding cassette and proton-antiport mechanisms in Arabidopsis. Plant Physiol. 2013;163: 1446-58.

75. Sakakibara H. Cytokinin biosynthesis and metabolism. In: Davies PJ, editor. The plant hormones: biosynthesis, signal transduction, action! 3rd ed. Dordrecht: Springer Netherlands; 2010. p. 95-114.

76. Hwang I, Sheen J, Müller B. Cytokinin signaling networks. Annu Rev Plant Biol. 2012:63:353-80.

77. Hirose N, Takei K, Kuroha T, Kamada-Nobusada T, Hayashi H, Sakakibara H. Regulation of cytokinin biosynthesis, compartmentalization and translocation. J Exp Bot. 2008;59:75-83.

78. Kudo T, Kiba T, Sakakibara H. Metabolism and long-distance translocation of cytokinins. J Integr Plant Biol. 2010;52:53-60.

79. Zhang K, Novak O, Wei Z, Gou M, Zhang X, Yu Y, et al. Arabidopsis ABCG14 protein controls the acropetal translocation of root-synthesized cytokinins. Nat Commun. 2014;5:3274.

80. Ko D, Kang J, Kiba T, Park J, Kojima M, Do J, et al. Arabidopsis ABCG14 is essential for the root-to-shoot translocation of cytokinin. Proc Natl Acad Sci U S A. 2014;111:7150-5.

81. Hirose N, Makita N, Yamaya T, Sakakibara H. Functional characterization and expression analysis of a gene, OsENT2, encoding an equilibrative nucleoside transporter in rice suggest a function in cytokinin transport. Plant Physiol. 2005;138:196-206

82. Sun J, Hirose N, Wang X, Wen P, Xue L, Sakakibara H, et al. Arabidopsis SOl33/AtENT8 gene encodes a putative equilibrative nucleoside transporter that is involved in cytokinin transport in planta. J Integr Plant Biol. 2005;47: 588-603.

83. Bürkle L, Cedzich A, Döpke C, Stransky H, Okumoto S, Gillissen B, et al. Transport of cytokinins mediated by purine transporters of the PUP family expressed in phloem, hydathodes, and pollen of Arabidopsis. Plant J. 2003; 34:13-26.

84. Zürcher E, Liu J, di Donato M, Geisler M, Müller B. Plant development regulated by cytokinin sinks. Science. 2016;353:1027-30.

85. Wulfetange K, Lomin SN, Romanov GA, Stolz A, Heyl A, Schmülling T. The cytokinin receptors of Arabidopsis are located mainly to the endoplasmic reticulum. Plant Physiol. 2011;156:1808-18.
86. Yamaguchi S. Gibberellin metabolism and its regulation. Annu Rev Plant Biol. 2008:59:225-51.

87. Gupta R, Chakrabarty SK. Gibberellic acid in plant: still a mystery unresolved. Plant Signal Behav. 2013;8:e25504.

88. Sun TP. Gibberellin-GID1-DELLA: a pivotal regulatory module for plant growth and development. Plant Physiol. 2010;154:567-70.

89. Tal I, Zhang Y, Jørgensen ME, Pisanty O, Barbosa ICR, Zourelidou M, et al. The Arabidopsis NPF3 protein is a GA transporter. Nat Commun. 2016;7:11486.

90. Kanno Y, Oikawa T, Chiba Y, Ishimaru Y, Shimizu T, Sano N, et al. AtSWEET13 and AtSWEET14 regulate gibberellin-mediated physiological processes. Nat Commun. 2016;7:13245.

91. Nour-Eldin HH, Andersen TG, Burow M, Madsen SR, Jorgensen ME, Olsen CE, et al. NRT/PTR transporters are essential for translocation of glucosinolate defence compounds to seeds. Nature. 2012;488:531-4.

92. Saito H, Oikawa T, Hamamoto S, Ishimaru Y, Kanamori-Sato M, SasakiSekimoto $Y$, et al. The jasmonate-responsive GTR1 transporter is required for gibberellin-mediated stamen development in Arabidopsis. Nat Commun. 2015;6:6095

93. Cook CE, Whichard LP, Turner B, Wall ME, Egley GH. Germination of witchweed (Striga lutea Lour.): isolation and properties of a potent stimulant. Science. 1966:154:1189-90.

94. Domagalska MA, Leyser O. Signal integration in the control of shoot branching. Nat Rev Mol Cell Biol. 2011;12:211-21.

95. Drummond RS, Janssen BJ, Luo Z, Oplaat C, Ledger SE, Wohlers MW, et al. Environmental control of branching in petunia. Plant Physiol. 2015;168:735-51.

96. Kretzschmar T, Kohlen W, Sasse J, Borghi L, Schlegel M, Bachelier JB, et al. A petunia $A B C$ protein controls strigolactone-dependent symbiotic signalling and branching. Nature. 2012:483:341-4.

97. Sasse J, Simon S, Gubeli C, Liu GW, Cheng X, Friml J, et al. Asymmetric localizations of the $A B C$ transporter PaPDR1 trace paths of directional strigolactone transport. Curr Biol. 2015;25:647-55.

98. Borghi L, Liu GW, Emonet A, Kretzschmar T, Martinoia E. The importance of strigolactone transport regulation for symbiotic signaling and shoot branching. Planta. 2016;243:1351-60.

99. Xie X, Wang G, Yang L, Cheng T, Gao J, Wu Y, et al. Cloning and characterization of a novel Nicotiana tabacum $A B C$ transporter involved in shoot branching. Physiol Plant. 2015;153:299-306.

100. Kohlen W, Charnikhova T, Liu Q, Bours R, Domagalska MA, Beguerie S, et al. Strigolactones are transported through the xylem and play a key role in shoot architectural response to phosphate deficiency in nonarbuscular mycorrhizal host Arabidopsis. Plant Physiol. 2011;155:974-87.

101. Waters MT, Gutjahr C, Bennett T, Nelson DC. Strigolactone signaling and evolution. Annu Rev Plant Biol. 2017;68:291-322.

102. Browse J. Jasmonate passes muster: a receptor and targets for the defense hormone. Annu Rev Plant Biol. 2009;60:183-205.

103. Wasternack C, Forner S, Strnad M, Hause B. Jasmonates in flower and seed development. Biochimie. 2013;95:79-85

104. Sato C, Aikawa K, Sugiyama S, Nabeta K, Masuta C, Matsuura H. Distal transport of exogenously applied jasmonoyl-isoleucine with wounding stress. Plant Cell Physiol. 2011;52:509-17.

105. Nguyen CT, Martinoia E, Farmer EE. Emerging jasmonate transporters. Mol Plant. 2017;10:659-61.

106. Ishimaru Y, Oikawa T, Suzuki T, Takeishi S, Matsuura H, Takahashi K, et al. GTR1 is a jasmonic acid and jasmonoyl---isoleucine transporter in Arabidopsis thaliana. Biosci Biotechnol Biochem. 2017;81:249-55.

107. Theodoulou FL, Job K, Slocombe SP, Footitt S, Holdsworth M, Baker A, et al. Jasmonic acid levels are reduced in COMATOSE ATP-binding cassette transporter mutants. Implications for transport of jasmonate precursors into peroxisomes. Plant Physiol. 2005;137:835-40.

108. Dave A, Hernández ML, He Z, Andriotis VME, Vaistij FE, Larson TR, et al. 12-oxo-phytodienoic acid accumulation during seed development represses seed germination in Arabidopsis. Plant Cell. 2011;23:583-99.

109. Park S, Gidda SK, James CN, Horn PJ, Khuu N, Seay DC, et al. The $a / \beta$ hydrolase CGI-58 and peroxisomal transport protein PXA1 coregulate lipid homeostasis and signaling in Arabidopsis. Plant Cell. 2013;25:1726-39.

110. Nyathi Y, De Marcos Lousa C, van Roermund CW, Wanders RJ, Johnson B, Baldwin SA, et al. The Arabidopsis peroxisomal ABC transporter, comatose, complements the Saccharomyces cerevisiae pxa1 pxa2Delta mutant for metabolism of long-chain fatty acids and exhibits fatty acyl-CoA-stimulated ATPase activity. J Biol Chem. 2010;285:29892-902. 
111. Baker A, Carrier DJ, Schaedler T, Waterham HR, van Roermund CW, Theodoulou FL. Peroxisomal ABC transporters: functions and mechanism. Biochem Soc Trans. 2015;43:959-65.

112. Mendiondo GM, Medhurst A, van Roermund CW, Zhang X, Devonshire J, Scholefield D, et al. Barley has two peroxisomal ABC transporters with multiple functions in $\beta$-oxidation. J Exp Bot. 2014;65:4833-47.

113. Li Q, Zheng J, Li S, Huang G, Skilling SJ, Wang L, et al. Transporter-mediated nuclear entry of jasmonoyl-isoleucine is essential for jasmonate signaling. Mol Plant. 2017;10:695-708.

114. Vlot AC, Dempsey DMA, Klessig DF. Salicylic acid, a multifaceted hormone to combat disease. Annu Rev Phytopathol. 2009;47:177-206.

115. Fragnière C, Serrano M, Abou-Mansour E, Métraux JP, L'Haridon F. Salicylic acid and its location in response to biotic and abiotic stress. FEBS Lett. 2011;585:1847-52.

116. Seyfferth C, Tsuda K. Salicylic acid signal transduction: the initiation of biosynthesis, perception and transcriptional reprogramming. Front Plant Sci. 2014:5:697.

117. Nawrath C, Métraux JP. Salicylic acid induction-deficient mutants of Arabidopsis express PR-2 and PR-5 and accumulate high levels of camalexin after pathogen inoculation. Plant Cell. 1999;11:1393-404.

118. Glazebrook J, Rogers EE, Ausubel FM. Isolation of Arabidopsis mutants with enhanced disease susceptibility by direct screening. Genetics. 1996;143:973-82

119. Serrano M, Wang B, Aryal B, Garcion C, Abou-Mansour E, Heck S, et al. Export of salicylic acid from the chloroplast requires the multidrug and toxin extrusion-like transporter EDS5. Plant Physiol. 2013;162:1815-21.

120. Dean JV, Mills JD. Uptake of salicylic acid 2-O-beta-D glucose into soybean tonoplast vesicles by an ATP-binding cassette transporter-type mechanism Physiol Plant. 2004;120:603-12

121. Dean JV, Mohammed LA, Fitzpatrick T. The formation, vacuolar localization, and tonoplast transport of salicylic acid glucose conjugates in tobacco cell suspension cultures. Planta. 2005;221:287-96.

122. Bleecker $A B$, Kende $H$. Ethylene: a gaseous signal molecule in plants. Annu Rev Cell Dev Biol. 2000;16:1-18

123. Shin K, Lee S, Song WY, Lee RA, Lee I, Ha K, et al. Genetic identification of ACC-RESISTANT2 reveals involvement of LYSINE HISTIDINE TRANSPORTER1 in the uptake of 1-aminocyclopropane-1-carboxylic acid in Arabidopsis thaliana. Plant Cell Physiol. 2015;56:572-82.

124. Pech J, Latché A, Bouzayen M. Ethylene biosynthesis. In: Davies PJ, editor. The Plant hormones: biosynthesis, signal transduction, action! 3rd ed. Dordrecht: Springer Netherlands; 2010. p. 115-36.

125. Kim TW, Wang ZY. Brassinosteroid signal transduction from receptor kinases to transcription factors. Annu Rev Plant Biol. 2010;61:681-704.

126. Fujioka S, Yokota T. Biosynthesis and metabolism of brassinosteroids. Annu Rev Plant Biol. 2003;54:137-64.

127. Symons GM, Reid JB. Brassinosteroids do not undergo long-distance transport in pea. Implications for the regulation of endogenous brassinosteroid levels. Plant Physiol. 2004;135:2196-206.

128. Symons GM, Ross JJ, Jager CE, Reid JB. Brassinosteroid transport. J Exp Bot. 2008;59:17-24

129. Turck F, Fornara F, Coupland G. Regulation and identity of florigen: FLOWERING LOCUS T moves center stage. Annu Rev Plant Biol. 2008;59: 573-94

130. Putterill J, Varkonyi-Gasic E. FT and florigen long-distance flowering control in plants. Curr Opin Plant Biol. 2016;33:77-82.

131. Corbesier L, Vincent C, Jang S, Fornara F, Fan Q, Searle I, et al. FT protein movement contributes to long-distance signaling in floral induction of Arabidopsis. Science. 2007;316:1030-3.

132. Liu L, Liu C, Hou X, Xi W, Shen L, Tao Z, et al. FTIP1 is an essential regulator required for florigen transport. PLoS Biol. 2012;10:e1001313.

133. Lindsey K, Casson S, Chilley P. Peptides: new signalling molecules in plants. Trends Plant Sci. 2002;7:78-83.

134. Hwang JU, Song WY, Hong D, Ko D, Yamaoka Y, Jang S, et al. Plant ABC transporters enable many unique aspects of a terrestrial plant's lifestyle. Mol Plant. 2016:9:338-55.

135. Galvan-Ampudia CS, Offringa R. Plant evolution: AGC kinases tell the auxin tale. Trends Plant Sci. 2007;12:541-7.

136. Cant N, Pollock N, Ford RC. CFTR structure and cystic fibrosis. Int J Biochem Cell Biol. 2014:52:15-25.

137. Kleine-Vehn J, Langowski L, Wisniewska J, Dhonukshe P, Brewer PB, Friml J. Cellular and molecular requirements for polar PIN targeting and transcytosis in plants. Mol Plant. 2008;1:1056-66.
138. Michniewicz M, Zago MK, Abas L, Weijers D, Schweighofer A, Meskiene I, et al. Antagonistic regulation of PIN phosphorylation by PP2A and PINOID directs auxin flux. Cell. 2007;130:1044-56.

139. Wang B, Bailly A, Zwiewka M, Henrichs S, Azzarello E, Mancuso S, et al. Arabidopsis TWISTED DWARF1 functionally interacts with auxin exporter ABCB1 on the root plasma membrane. Plant Cell. 2013;25:202-14.

140. Wu G, Otegui MS, Spalding EP. The ER-localized TWD1 immunophilin is necessary for localization of multidrug resistance-like proteins required for polar auxin transport in Arabidopsis roots. Plant Cell. 2010;22:3295-304.

141. Dharmasiri S, Swarup R, Mockaitis K, Dharmasiri N, Singh SK, Kowalchyk M, et al. AXR4 is required for localization of the auxin influx facilitator AUX1. Science. 2006;312:1218-20.

142. Mao H, Nakamura M, Viotti C, Grebe M. A framework for lateral membrane trafficking and polar tethering of the PEN3 ATP-binding cassette transporter. Plant Physiol. 2016;172:2245-60.

143. Park Y, Xu ZY, Kim SY, Lee J, Choi B, Lee J, et al. Spatial regulation of $A B C G 25$, an $A B A$ exporter, is an important component of the mechanism controlling cellular ABA levels. Plant Cell. 2016;28:2528-44.

144. Paciorek T, Zazimalova E, Ruthardt N, Petrasek J, Stierhof YD, Kleine-Vehn J, et al. Auxin inhibits endocytosis and promotes its own efflux from cells. Nature. 2005:435:1251-6.

145. Zourelidou M, Absmanner B, Weller B, Barbosa IC, Willige BC, Fastner A, et al. Auxin efflux by PIN-FORMED proteins is activated by two different protein kinases, D6 PROTEIN KINASE and PINOID. Elife. 2014;3:e02860.

146. Henrichs S, Wang B, Fukao Y, Zhu J, Charrier L, Bailly A, et al. Regulation of ABCB1/PGP1-catalysed auxin transport by linker phosphorylation. EMBO J. 2012;31:2965-80.

147. Christie JM, Yang H, Richter GL, Sullivan S, Thomson CE, Lin J, et al. phot1 inhibition of $A B C B 19$ primes lateral auxin fluxes in the shoot apex required for phototropism. PLoS Biol. 2011;9:e1001076.

148. Ishimaru Y, Washiyama K, Oikawa T, Hamamoto S, Uozumi N, Ueda M Dimerization of GTR1 regulates their plasma membrane localization. Plant Signal Behav. 2017;12(16):e1334749.

149. Geisler M, Blakeslee JJ, Bouchard R, Lee OR, Vincenzetti V, Bandyopadhyay A, et al. Cellular efflux of auxin catalyzed by the Arabidopsis MDR/PGP transporter AtPGP1. Plant J. 2005;44:179-94.

150. Bailly A, Yang H, Martinoia E, Geisler M, Murphy AS. Plant lessons: exploring ABCB functionality through structural modeling. Front Plant Sci. 2012;2:108.

151. Hayashi M, Nito K, Takei-Hoshi R, Yagi M, Kondo M, Suenaga A, et al. Ped3p is a peroxisomal ATP-binding cassette transporter that might supply substrates for fatty acid $\beta$-oxidation. Plant Cell Physiol. 2002;43:1-11.

152. Ito $H$, Gray WM. A gain-of-function mutation in the Arabidopsis pleiotropic drug resistance transporter PDR9 confers resistance to auxinic herbicides. Plant Physiol. 2006;142:63-74

153. Kim DY, Bovet L, Maeshima M, Martinoia E, Lee $Y$. The ABC transporter AtPDR8 is a cadmium extrusion pump conferring heavy metal resistance. Plant J. 2007:50:207-18.

154. Cheng Y, Zhao Y. A role for auxin in flower development. J Integrative Plant Biol. 2007:49:99-104. 